CLINICAL STUDY

\title{
Variation in the bone morphogenetic protein-2 gene: effects on fat and lean body mass in young and elderly women
}

\author{
Fiona McGuigan ${ }^{1,2}$, Emma Larzenius ${ }^{1,2}$, Mattias Callreus ${ }^{1,2}$, Paul Gerdhem ${ }^{1,3}$, Holger Luthman ${ }^{4}$ \\ and Kristina Akesson ${ }^{1,2}$ \\ ${ }^{1}$ Clinical and Molecular Osteoporosis Research Unit, Department of Clinical Sciences Malmö, Lund University, 205 02 Malmö, Sweden, ${ }^{2}$ Department of \\ Orthopaedics, Malmö University Hospital, 20502 Malmö, Sweden, ${ }^{3}$ Department of Orthopaedics, Karolinska University Hospital, S-171 76 Stockholm, \\ Sweden and ${ }^{4}$ Medical Genetics Unit, Department of Clinical Sciences Malmö, Lund University, 20520 Malmö, Sweden \\ (Correspondence should be addressed to K Åkesson; Email: kristina.akesson@med.lu.se)
}

\begin{abstract}
Objective: Bone morphogenetic protein-2 (BMP2) plays a critical role in osteoblastogenesis and adipogenesis from osteoprogenitor cells. The balance between osteogenic and adipogenic effects is influenced by BMP2 concentration, transcription factors and age. BMP2 single nucleotide polymorphisms (SNPs) may contribute to osteoporosis risk, but the relationship between adiposity and body composition has not been explored. We investigated the relationship between BMP2 polymorphisms and body composition in young and elderly women.

Design: Population-based association study.

Methods: Four BMP2 SNPs studied. Total body fat and lean mass measured by DEXA in two cohorts: 'PEAK-25' women aged $25( \pm 0.00)(n=993)$ and osteoporosis prospective risk assessment (OPRA) women aged $75( \pm 0.00)$ years $(n=1001)$.

Results: We found no association between BMP2 SNPs and fat or lean mass, however, we observed consistent although non-significant trends. Polymorphisms, rs235767 and Ser37Ala, exerted opposing effects on most parameters of soft tissue and bone mass in both cohorts. This relationship appeared to be age specific with large differences between alleles observed (fat mass; Ser37Ala: 14.3\% (PEAK-25), $-3.5 \%$ (OPRA)). These initial results appear to suggest that alleles exerting a beneficial effect in young women may subsequently contribute to phenotypes associated with osteoporosis risk in elderly women. Conclusions: While further analyses in other comparative populations are necessary, in this study of almost 2000 women we observed interesting, although non-significant trends, regarding the effects of variation in the BMP2 gene on parameters of body mass. Although the exact nature of the relationship remains uncertain, we suggest that the mechanisms are influenced by age and environmental factors.
\end{abstract}

European Journal of Endocrinology 158 661-668

\section{Introduction}

Osteoporosis is a complex multifactorial disease comprising environmental and genetic components. Body mass index (BMI) is a strong predictor of bone mineral density (BMD) and bone loss (1). An inverse relationship exists between the two such that overweight individuals have a higher BMD and lower risk of fracture than those of average weight (2).

Both the fat and lean mass components of soft tissue influence bone mass (3). At its simplest level, the association between body mass and BMD may be mediated through the effects of skeletal loading and via the action of oestrogen, which is metabolised in peripheral adipose tissue (4). More recently, the complexity of the relationship between fat and bone has been recognised. Osteoblasts and adipocytes share a common mesenchymal stem cell lineage with a degree of plasticity between them (5), depending on environmental and physiological signals (6-8). Evidence suggests both shared and individual genes contribute to the overlapping phenotypes of BMD and body weight (9). One such pleiotropic candidate is the bone morphogenetic protein-2 (BMP2), which is implicated in both osteoblastogenesis and adipogenesis from common progenitor cells. The pathway favoured depends on complex interactions including BMP2 concentration and several other regulators (10). Evidence from in vitro studies also suggests that muscle satellite cells are able to differentiate into osteoblasts following BMP2 exposure (11).

BMP2 gene variants have variously been linked to $\mathrm{BMD}$ and osteoporotic fracture, and in our cohorts with ultrasound parameters (12). Since adipocytes may be influenced by BMP2, we hypothesised that variation in BMP2 might contribute to the distribution of fat mass and hence indirectly contribute to bone mass. To investigate the potential relationship between BMP2 gene variants and body composition, we used two 
population-based Swedish cohorts, the PEAK-25 study of young women and the OPRA study of elderly women.

\section{Materials and methods}

\section{Subjects}

The study subjects were Caucasian participants in one of two population-based studies. The Malmö Osteoporosis Prospective Risk Assessment (OPRA) study consists of women all aged 75 years, collected between 1995 and 1999. Women were invited to follow-up visits 1,3 and 5 years after inclusion and we report here on measurements made at the 5-year follow-up visit.

The 'PEAK-25' cohort consists of young women living in Malmö, Sweden, between 1999 and 2003. The women, all of whom were aged 25 years at inclusion, were randomly selected from the city files. Full details of both cohorts have been reported previously $(12,13)$. All participants gave informed consent and the studies were approved by the Lund University Ethics Committee.

\section{Measurements}

Total body scans from dual-energy X-ray absorptiometry (PEAK-25: Lunar Prodigy, OPRA: Lunar DPX-L (Lunar Corporation, Madison, WI, USA)) were used to analyse body composition; fat mass (g), lean mass (g) and BMD $\left(\mathrm{g} / \mathrm{cm}^{2}\right)$. Lumbar spine (LS) and femoral neck (FN) BMD were also measured. The participants included in the reported analysis comprised $n=1001$ (OPRA) and $n=993$ (PEAK-25), for whom a DXA measurement and BMP2 genotype result was available.

\section{BMP2 SNPs and genotyping}

In addition to the potentially functional Ser37Ala SNP (rs2273073) originally implicated in low BMD, we selected an additional three SNPs to encompass the gene and its surroundings (http://www.ensembl.org). In the absence of available data on tagging SNPs at that time, SNPs were selected on the following criteria: relative position to the gene, minor allele frequency in Caucasian populations and distance between the polymorphisms. These were (in chromosomal order) rs235710 (10 kb upstream), rs2273073 (Ser37Ala) in exon 2, rs235767 in intron 2 and rs235754 (8 kb downstream) (12).

The polymorphisms were all genotyped blind by the $5^{\prime}$ nuclease Taqman assay (Applied Biosystems, Foster City, California, USA) with $1 \%$ routinely repeated to ensure agreement of results. A further 3\% of all PEAK-25 samples and $4.5 \%$ of all OPRA samples underwent additional checks for the Ser37Ala polymorphism, with concurrence of results. Full details of the methodology have been described previously (12).

\section{Statistical analysis}

Statistical analyses were performed using SPSS v14 (SPSS Inc., Chicago). Hardy-Weinberg equilibrium (HWE) was calculated by $\chi^{2}$-test. Linkage disequilibrium between SNPs (Lewontin's $D^{\prime}$ and $r^{2}$ ) was calculated using the EH, $2 \mathrm{BY} 2$ (14) and Haploview programs. Haplotypes were derived from Ser37Ala and rs235767 using PHASE v2.02 (15). Two common haplotypes accounted for $>98 \%$ of alleles at the BMP 2 locus: haplotype 1 'Ser/A' $(61.5 \%)$ and haplotype 2 'Ser/C' (36.5\%). Haplotype analyses were performed, according to whether individuals possessed zero, one or two copies of the haplotype.

Non-normally distributed variables were log transformed prior to analysis $(<0.95$, Shapiro-Wilk). Genotype-specific differences in variables were investigated using GLM-ANOVA, correcting for confounders including height, oestrogen use, years since menarche and smoking, but not age, since all individuals within each cohort were of the same age (PEAK-25: 25.5 years, range 25.03-25.99 and OPRA: 75.2 years, range 75.0-75.8). Multiple regression analysis determined independent predictors of fat and lean mass, using a model containing genotype or haplotype, height, smoking, age at menarche and contraceptive pill use as variables. The $P$ values are presented uncorrected. Significance was not retained when the adjusted level of significance equivalent to $P=0.05$, based on a modified Bonferroni correction, was applied.

\section{Results}

All genotype frequencies were in apparent HWE (Table 1). For rs 235767, there was an under-representation of the 'AA' genotype in the young when compared with the elderly cohort of women; however, the allele frequencies in both populations are largely similar (A/C: PEAK-25 61\%/ $39 \%$, OPRA $\left.63 \% / 37 \% ; \chi^{2}=5.9 ; P=0.05\right)$. Neither cohort displayed any genotype-related differences in the general clinical characteristics.

The baseline clinical characteristics of the two study populations are shown in Table 2 and were significantly different between the cohorts. Compared with the older women, the 25-year olds were taller and weighed less. Not unexpectedly they had more lean $(\sim 8 \%)$ and less fat $(25 \%)$ tissue mass, which is reflected in the lower ratio of fat to lean mass. Smoking incidence was higher in the young women (26\% vs $13.8 \%$ ) and the age of onset of menarche was younger (12.7 vs 14.0 years). There were no genotype-related differences in height, weight or BMI for either cohort.

As expected, the correlations between measures of body composition and total body BMD were substantial for both cohorts (range, $r=0.35-0.53$ ) as was the 
Table 1 Bone morphogenetic protein-2 (BMP2) genotype frequencies, Hardy-Weinberg equilibrium (HWE) and distributions between the cohorts.

\begin{tabular}{lccc}
\hline & \multicolumn{2}{c}{ Genotype frequency $(\%)$} & \\
\cline { 2 - 3 } SNP & PEAK-25 & OPRA & $\begin{array}{c}\text { Between- } \\
\text { cohorts }\end{array}$ \\
\hline rs235710 (G/A) & & & \\
GG & 28.1 & 27.6 & \\
GA & 49.7 & 48.0 & $P=0.40$ \\
AA & 21.6 & 24.1 & \\
HWE $(P=)$ & 0.86 & 0.26 & \\
rs2373073 (T/G) & & & \\
TT & 96.2 & 97.0 & \\
TG & 3.4 & 3.0 & $P=0.27$ \\
GG & 0.2 & 0.0 & \\
HWE $(P=)$ & 0.05 & 0.63 & \\
rs235767 (A/C) & & & \\
AA & 35.8 & 40.7 & \\
AC & 49.9 & 45.0 & \\
CC & 14.3 & 14.4 & \\
HWE $(P=)$ & 0.12 & 0.28 & \\
rs235754 $(G / C)$ & & & \\
GG & 26.6 & 28.8 & \\
GC & 49.8 & 49.8 & $P=0.38$ \\
CC & 23.3 & 21.1 & \\
HWE $(P=)$ & 0.97 & 0.87 & \\
\hline
\end{tabular}

correlation between fat mass and lean mass. In OPRA, the correlation between lean mass and total body BMD was lower than in the young women while the correlation with fat mass was considerably higher (Table 2).

Table 2 Clinical characteristics of the PEAK-25 and osteoporosis prospective risk assessment (OPRA) study populations.

\begin{tabular}{lcc}
\hline & $\begin{array}{c}\text { PEAK-25 } \\
(n=993)\end{array}$ & $\begin{array}{c}\text { OPRA } \\
(n=1001)\end{array}$ \\
\hline Age (years) & $25.5 \pm 0.2$ & $75.2 \pm 0.1$ \\
Height (cm) & $167.6 \pm 6.1$ & $160.6 \pm 5.8$ \\
Weight (kg) & $64.6 \pm 11.0$ & $67.8 \pm 11.5$ \\
BMl (kg/m $\left.{ }^{2}\right)$ & $22.9 \pm 3.7$ & $26.3 \pm 4.2$ \\
Age at menarche (years) & $12.7 \pm 1.3$ & $14.0 \pm 1.4$ \\
Age at menopausal (years) & $\mathrm{N} / \mathrm{A}$ & $49.1 \pm 4.9$ \\
Current smokers & $258(26.0 \%)$ & $138(13.8 \%)$ \\
Total body BMD (g/cm $\left.{ }^{2}\right)$ & $1.478 \pm 0.07$ & $1.010 \pm 0.09$ \\
Total body lean mass $(\mathrm{g})$ & $40406 \pm 4658$ & $37326 \pm 3936$ \\
Total body fat mass (g) & $21046 \pm 8236$ & $28119 \pm 7885$ \\
Fat mass (\% of soft tissue) & $31.7 \%$ & $40.3 \%$ \\
Lean mass (\% of soft tissue) & $63.4 \%$ & $55.1 \%$ \\
Ratio fat:lean mass & $0.52 \pm 0.19$ & $0.69 \pm 0.15$ \\
Change since base line & $\mathrm{N} / \mathrm{A}$ & $-1.43 \pm 1.6$ \\
Height (cm) & $\mathrm{N} / \mathrm{A}$ & $-1.71 \pm 5.1$ \\
Weight (kg) & $\mathrm{N} / \mathrm{A}$ & $-625 \pm 4164$ \\
Fat mass (g) & $\mathrm{N} / \mathrm{A}$ & $-851 \pm 1688$ \\
Lean mass (g) & & \\
Correlation: body composition & & \\
$\quad$ and BMD & 0.35 & 0.53 \\
Total body BMD and fat mass & 0.45 & 0.36 \\
Total body BMD and lean & & \\
mass & 0.33 & 0.43 \\
Fat mass and lean mass & & \\
\hline
\end{tabular}

Values are: Mean \pm s.D. or number (\%) or \%. Between-cohort comparison of variables, all $P$ values $<0.05$.
In both the PEAK-25 and OPRA cohorts, there was no significant association with fat mass for either genotypes or haplotypes, neither could we detect any differences in the fat-lean mass ratio. Categorising individuals according to whether they fell into the lowest or highest quintile of BMI did not appreciably alter the results.

In the young women, regression analysis identified rs235767 as a predictor of lean mass $(P=0.008)$ although this decreased to $P=0.06$ when height was additionally included in the model. Although individuals carrying one or two copies of the variant $C$ allele had incrementally lower values, the association was not significant. Individuals carrying two copies of the common haplotype 1 (Ser-A) had the highest lean mass values when compared with one copy (intermediate) and zero copies (lowest), $P=0.04$. As before, when height was taken into consideration, the relationship was abolished. The data did not alter appreciably when BMI category was taken into consideration. Haplotype 2 (Ser-C) was a non-significant predictor of lean mass $(P=0.081)$ and values decreased with haplotype copy number.

In the elderly women, BMP2 genotype was not an independent predictor of lean mass at baseline although a borderline association was identified between rs235767 and lean mass $(P=0.052)$ at follow-up. Individuals who were homozygous for the variant allele had higher lean mass at both time points but this was not incremental and was not significant either before or after correction. There were no significant genotype differences for the ratios of fat to lean mass or weight change between 20 and 75 years. Analysing only those in the highest and lowest quintiles of BMI did not appreciably alter the results. Exclusion of 69 women with reported diabetes (based on the known effects on adipose tissue from diabetes) gave essentially the same results. Regression analysis identified haplotype 1 as an independent predictor of lean mass at follow-up $(P=0.037)$ but not baseline. Similarly, haplotype 2 had a non-significant trend $(P=0.08)$. Although nonsignificant, individuals carrying one or two copies of haplotype 1 had the lowest values but not in a dosedependent manner, while individuals carrying one or two copies of haplotype 2 had the highest values and a gene dose effect was observed.

\section{SNP and age effects on body composition}

SNP rs235767 showed consistent dose dependent, although non-significant trends in the direction of effect across the parameters measured. For BMI, weight, lean mass, fat mass and BMD at all sites, 'CC' homozygotes had the lowest values for all measures (Fig. 1A PEAK-25). SNP Ser37Ala also showed consistent nonsignificant trends of effect, however, in this instance the Ala/Ala homozygotes, tended to display the highest mean values for the measures of body composition and 

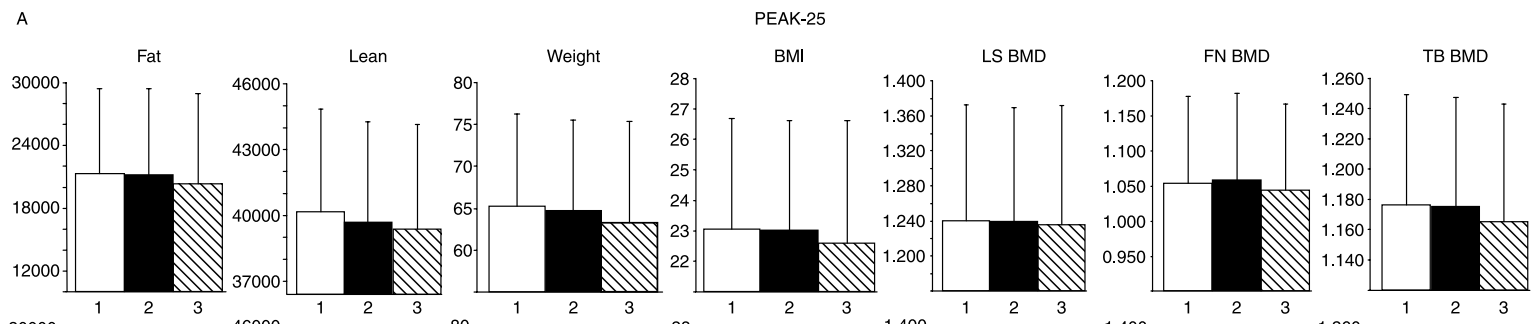

rs235767
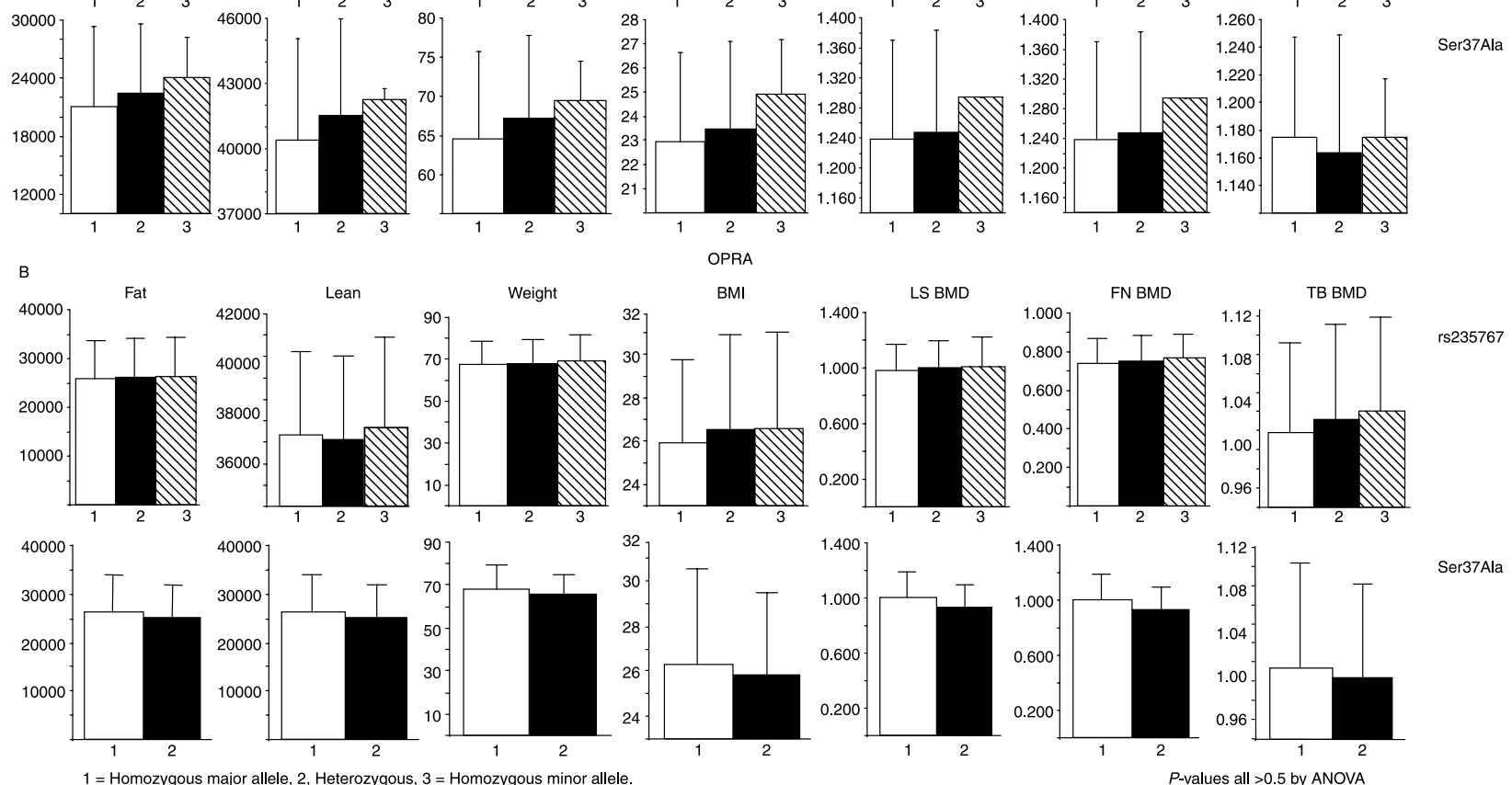

Ser37Ala

Figure 1 ( $A$ and $B$ ) Non-significant trends of the BMP2 genotype effect of SNP rs235767 and Ser37Ala on body composition parameters in young and elderly women. BMD values for total body, spine and hip are included for comparison. Values are mean \pm S.D.

BMD at the spine (Fig. 1A). (For the other SNPs, the relationship between genotype and phenotype was more variable (data not shown)).

Individuals with two copies of haplotype 1 tended to have the highest values for all the parameters measured, while individuals with two copies of haplotype 2 tended to have the lowest values. In both instances, the response was dose dependent (data not shown).

As in the young women, rs235767 showed consistently similar, non-significant trends in the direction of effect across the parameters measured at baseline. It is interesting to note, however, that the direction of the effect is the opposite of what is seen in the younger women, i.e. the homozygous variant individuals tended to have the highest mean values. With the exception of lean mass, the relationship was dose dependent (Fig. 1B). As far as can be compared, given the absence of homozygotes for the rare 'Ala' allele in the OPRA cohort, the Ser37Ala trends, as observed in the young women, were the opposite of those for rs235767 (Fig. 1B OPRA). Individuals with two copies of haplotype 1 tended to have the lowest values and individuals with two copies of haplotype 2 tended to have the highest values (data not shown).

Although it is not possible to make direct comparisons between the young and older women, the results suggest that in young adulthood, SNP Ser37Ala seems to be associated with higher fat and lean mass levels (for heterozygotes, $\sim 7 \%$ greater fat and $3 \%$ greater lean mass compared with 'Ser/Ser' homozygotes) and higher BMD levels. In old age, this relationship is reversed and the variant allele is associated with reduced fitness in terms of these measurements (Fig. 2A and B).

Five years after the baseline measurement for Ser37Ala, the observed trends are essentially the same as baseline for all the parameters measured. Similarly for rs 235767 with the exception of fat mass, where the rare 'CC' homozygotes display the lowest rather than the highest values. Figure 3 shows the change in weight, lean mass and fat mass in the OPRA cohort between the baseline measurement and follow-up $\sim 5$ years later. It is apparent that in very elderly women, rs235767 'CC' homozygotes and Ser37Ala heterozygotes loose more fat mass than lean mass during this period compared with the other genotypes. 


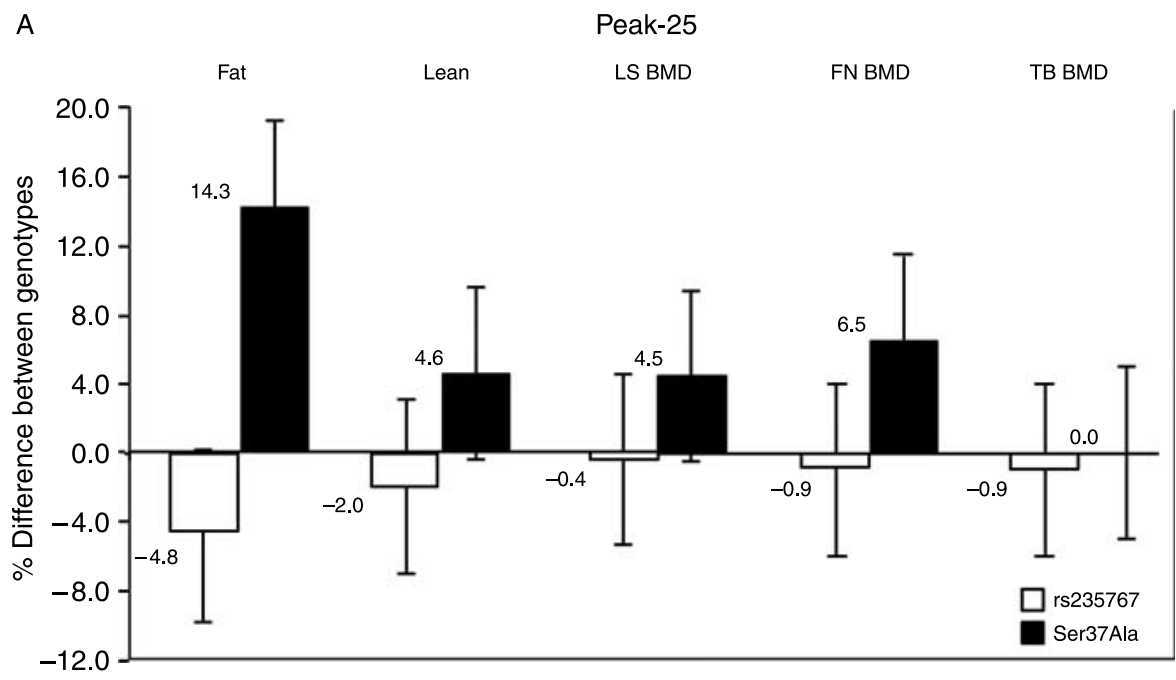

B

OPRA

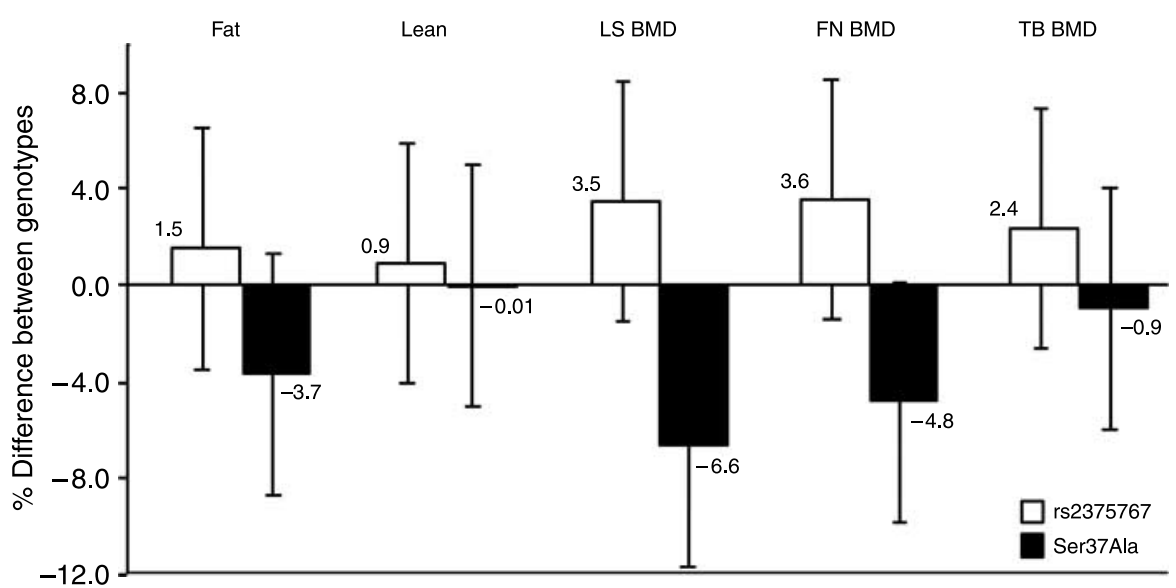

Figure 2 ( $A$ and $B$ ) Comparison of BMP2 genotype effects from SNPs rs235767 and Ser37Ala on body composition parameters in young and elderly women, and using percentage values to show the differential effects between the major and minor alleles. BMD values for total body, spine and hip are included for comparison. Values are mean and $95 \% \mathrm{Cl}$. (A) Values are the \% difference between major allele and minor allele homozygotes for both SNPs. (B) Values are the \% difference between major allele and minor allele homozygotes for rs235767 and between major allele homozygotes and heterozygotes or Ser37Ala.

\section{Discussion}

Evidence in the literature for the relative importance of the contributions of fat and lean mass to bone mineral density is inconsistent, perhaps reflecting discrepancies in the ages of the populations studied. In young women, lean mass is believed to be the major determinant of BMD while in older women fat mass has a larger impact (16). With increasing age, there is a general decline in muscle mass and a concomitant increase in adiposity contributing to frailty and reduced fitness.

Several genes that have been studied in relation to BMD, e.g. VDR, ER and LRP5, have pleiotropic effects and are implicated in a range of diseases including obesity and insulin resistance (17). The BMP2 gene has received some attention with regard to BMD $(18,19)$, however, despite a role in adipocyte differentiation both in vitro and in vivo (10) it has not yet been studied with regard to body composition. In the present study, we have investigated the relationship between variation in the BMP2 gene and the components of body composition: fat and lean mass in 25- and 75-year-old women.

Our results indicate that while BMP2 genotype is not significantly associated with absolute measures of fat mass, lean mass (or indeed bone mass), there is nevertheless a possible, albeit complex relationship between them which appears to be altered by age and possibly by other genetic and environmental interactions. 


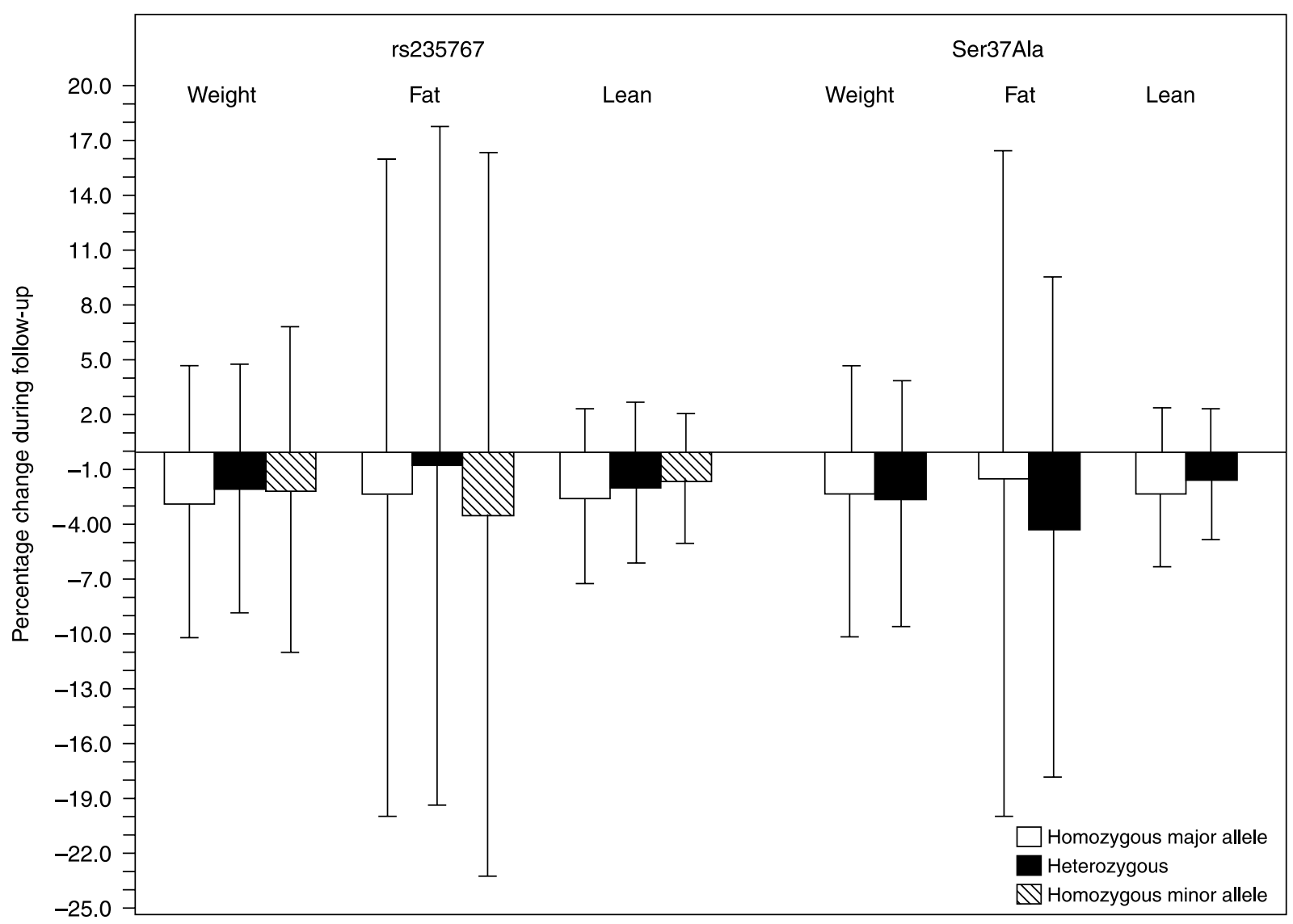

Figure 3 BMP2 genotype effects from SNPs rs235767 and Ser37Ala on percentage change in weight, lean and fat mass between baseline and follow-up visits in elderly women. Values for each genotype are mean $\%$ change during follow-up period, error bars are \pm 1 standard deviation.

Two polymorphisms, rs235767 and the potentially functional Ser37Ala, exerted opposing effects on almost all the parameters of soft tissue and bone mass measured in both cohorts of women. We also observed that alleles that could be considered beneficial to bone mass in the young women subsequently contributed to phenotypes associated with osteoporosis risk in the elderly women. Opposing effects of polymorphisms, such as we have observed, have previously been reported for PPAR- $\gamma$ (20) and MTHFR genotypes (21); however, we are unaware of other studies that have reported, as we do, age-related reversal of the relationship between genotype and phenotype. We cannot fully explain our observations in the context of the present study. The results could have arisen simply by chance - replication in comparative populations is necessary to refute or confirm our findings. In the absence of detailed dietary information in these cohorts, we also cannot exclude the possibility that small differences in BMI could have been contributed to by differences in the diet.

It could, however, reflect age-related environmental exposures, given that there are 55-60 years between the childhood and young adolescence of the two cohorts, or gene-microenvironment interactions. An alternative explanation could be antagonistic pleiotropy (whereby genes, advantageous during times of reproductive activity have harmful effects later in life), which is thought to be uncommon in humans, but has been observed in animal models of reproduction, immunity and body composition (22). The literature provides evidence to suggest that our findings are not the result of statistical anomaly. BMPs control stem cell commitment to various lineages and in vitro studies indicate that adipocyte differentiation depends on BMP2 dosage, precursor cell type and cell stage (23, 24) and the presence of other regulators such as Schnurri-2 and PPAR- $\gamma(10,25,26)$. The balance between osteogenic and adipogenic effects mediated by BMP2 appear to be reciprocal and influenced by ageing, such that ageing activates adipogenic and suppresses osteogenic characters in stem cells (27-29) an observation that may contribute indirectly to the explanation of our findings.

Twin studies indicate that the similarity between monozygotic twins diminish with age, suggesting that 
gene penetrance is susceptible to external influences $(30,31)$. By studying two cohorts from opposite ends of the age spectrum, we have identified relationships that might have otherwise been overlooked as a methodological artefact between individually published studies and therein lies one of the strengths of our study. The narrow age range and similar ethnic background of our cohorts also eliminate at least some of the confounding variation. Our data also highlight the importance of considering the effect of gene variation, not as static, but in the context of constantly changing interactions with the microenvironment. A weakness in our study is that at the time when genotyping was completed, no functional SNPs apart from Ser37Ala had been identified. Despite having chosen our SNPs to broadly encompass the gene region and inclusion of the potentially functional Ser37Ala polymorphism, it is not possible to capture all variation within the gene. However, $76 \%$ of the genetic variation in the locus is represented by five possible haplotypes identified in our population and inclusion of tag SNPs from the conserved haplotype block covering the gene, would have captured only an additional 15\%. Until replication studies have been performed in comparative agedistributed populations; however, we should be cautious in the conclusions we draw from these potentially interesting observations.

To conclude, in this study consisting of two distinct populations of almost 2000 women, we suggest that polymorphisms of the BMP 2 gene may be linked with measures of body composition, fat and lean mass. Interestingly, the effects are not uniform, indicating that age and environmental factors may play important roles through mechanisms that are not possible to fully explore in the current setting.

\section{Acknowledgements}

Support for the study was received from the Swedish Research Council, Greta and Johan Kock Foundation, A Påhlsson Foundation, A Osterlund Foundation, Swedish Center for Sports Medicine Research, Malmö University Hospital Research Foundation and the Swedish Medical Society. Thanks are also extended to Lisa Jansson for genotyping support and the research nurses at the Clinical and Molecular Osteoporosis Research Unit, and to all the women who kindly participated in the study.

\section{References}

1 Edelstein SL \& Barrett-Connor E. Relation between body size and bone mineral density in elderly men and women. American Journal of Epidemiology 1993138 160-169.

2 De Laet C, Kanis JA, Oden A, Johanson H, Johnell O, Delmas P, Eisman JA, Kroger H, Fujiwara S, Garnero P, McCloskey EV,
Mellstrom D, Melton LJ, III, Meunier PJ, Pols HA, Reeve J, Silman A $\&$ Tenenhouse A. Body mass index as a predictor of fracture risk: a meta-analysis. Osteoporosis International 200516 1330-1338.

3 Reid IR. Relationships among body mass, its components, and bone. Bone 200231 547-555.

4 Simpson E, Rubin G, Clyne C, Robertson K, O’Donnell L, Jones M \& Davis $\mathrm{S}$. The role of local estrogen biosynthesis in males and females. Trends in Endocrinology and Metabolism 200011 184-188.

5 Prockop DJ. Marrow stromal cells as stem cells for nonhematopoietic tissues. Science 1997276 71-74.

6 Beresford JN, Bennett JH, Devlin C, Leboy PS \& Owen ME. Evidence for an inverse relationship between the differentiation of adipocytic and osteogenic cells in rat marrow stromal cell cultures. Journal of Cell Science 1992102 341-351.

7 Nuttall ME \& Gimble JM. Controlling the balance between osteoblastogenesis and adipogenesis and the consequent therapeutic implications. Current Opinion in Pharmacology $20044290-294$.

8 Pittenger MF, Mackay AM, Beck SC, Jaiswal RK, Douglas R, Mosca JD, Moorman MA, Simonetti DW, Craig S \& Marshak DR. Multilineage potential of adult human mesenchymal stem cells. Science 1999284 143-147.

9 Nguyen TV, Howard GM, Kelly PJ \& Eisman JA. Bone mass, lean mass, and fat mass: same genes or same environments? American Journal of Epidemiology 1998147 3-16.

10 Jin W, Takagi T, Kanesashi SN, Kurahashi T, Nomura T, Harada J \& Ishii S. Schnurri-2 controls BMP-dependent adipogenesis via interaction with Smad proteins. Developmental Cell 200610 461-471.

11 Wada MR, Inagawa-Ogashiwa M, Shimizu S, Yasumoto S \& Hashimoto N. Generation of different fates from multipotent muscle stem cells. Development 2002129 2987-2995.

12 McGuigan FE, Larzenius E, Callreus M, Gerdhem P, Luthman H \& Åkesson K. Variation in the BMP2 gene: bone mineral density and ultrasound in young adult and elderly women. Calcified Tissue International $200781254-262$.

13 Gerdhem P, Brandstrom H, Stiger F, Obrant K, Melhus H, Ljunggren $\mathrm{O}$, Kindmark A \& Akesson K. Association of the collagen type 1 (COL1A 1) Sp1 binding site polymorphism to femoral neck bone mineral density and wrist fracture in 1044 elderly Swedish women. Calcified Tissue International $2004 \mathbf{7 4}$ 264-269.

14 Terwilliger JD \& Ott J. Handbook of Human Genetic Linkage Baltimore, MA: Johns Hopkins University Press. Baltimore, USA 1994.

15 Stephens M, Smith NJ \& Donnelly P. A new statistical method for haplotype reconstruction from population data. American Journal of Human Genetics 200168 978-989.

16 Di Monaco M, Vallero F, Di Monaco R, Tappero R \& Cavanna A. Skeletal muscle mass, fat mass, and hip bone mineral density in elderly women with hip fracture. Journal of Bone and Mineral Metabolism 200725 237-242.

17 Ferrari SL \& Rizzoli R. Gene variants for osteoporosis and their pleiotropic effects in aging. Molecular Aspects of Medicine 200526 145-167.

18 Medici M, van Meurs JB, Rivadeneira F, Zhao H, Arp PP, Hofman A, Pols HA \& Uitterlinden AG. BMP-2 gene polymorphisms and osteoporosis: the Rotterdam Study. Journal of Bone and Mineral Research 200621 845-854.

19 Styrkarsdottir U, Cazier JB, Kong A, Rolfsson O, Larsen H, Bjarnadottir E, Johannsdottir VD, Sigurdardottir MS, Bagger Y, Christiansen C, Reynisdottir I, Grant SF, Jonasson K, Frigge ML, Gulcher JR, Sigurdsson G \& Stefansson K. Linkage of osteoporosis to chromosome 20p12 and association to BMP2. PLoS Biology 20031 E69.

20 Cecil JE, Watt P, Palmer CN \& Hetherington M. Energy balance and food intake: the role of PPARgamma gene polymorphisms. Physiology and Behavior $2006 \mathbf{8 8} 227-233$.

21 Parle-McDermott A, Mills JL, Molloy AM, Carroll N, Kirke PN, Cox C, Conley MR, Pangilinan FJ, Brody LC \& Scott JM. The MTHFR1298CC and 677TT genotypes have opposite associations with red cell folate levels. Molecular Genetics and Metabolism 200688 290-294. 
22 Fernandez B, Garcia-Dorado A \& Caballero A. The effect of antagonistic pleiotropy on the estimation of the average coefficient of dominance of deleterious mutations. Genetics $2005 \mathbf{1 7 1}$ 2097-2112.

23 Zhao GQ. Consequences of knocking out BMP signaling in the mouse. Genesis 200335 43-56.

24 Varga AC \& Wrana JL. The disparate role of BMP in stem cell biology. Oncogene 200524 5713-5721.

25 Hata K, Nishimura R, Ikeda F, Yamashita K, Matsubara T, Nokubi T \& Yoneda T. Differential roles of Smad1 and p38 kinase in regulation of peroxisome proliferator-activating receptor gamma during bone morphogenetic protein 2-induced adipogenesis. Molecular Biology of the Cell $200314545-555$.

26 Rosen CJ \& Bouxsein ML. Mechanisms of disease: is osteoporosis the obesity of bone? Nature Clinical Practice. Rheumatology 20062 35-43.

27 Justesen J, Stenderup K, Ebbesen EN, Mosekilde L, Steiniche T \& Kassem M. Adipocyte tissue volume in bone marrow is increased with aging and in patients with osteoporosis. Biogerontology 2001 2 165-171.
28 Moerman EJ, Teng K, Lipschitz DA \& Lecka-Czernik B. Aging activates adipogenic and suppresses osteogenic programs in mesenchymal marrow stroma/stem cells: the role of PPARgamma2 transcription factor and TGF-beta/BMP signaling pathways. Aging Cell 20043 379-389.

29 Tseng TH \& He TC. Bone morphogenetic proteins and adipocyte differentiation. Cell Science Reviews 20073 1-16.

30 Pocock NA, Eisman JA, Hopper JL, Yeates MG, Sambrook PN \& Eberl S. Genetic determinants of bone mass in adults. A twin study. Journal of Clinical Investigation $1987 \mathbf{8 0} 706-710$.

31 Smith DM, Nance WE, Kang KW, Christian JC \& Johnston CC, Jr. Genetic factors in determining bone mass. Journal of Clinical Investigation $1973 \mathbf{5 2} 2800-2808$.

Received 5 February 2008

Accepted 6 February 2008 